\title{
Optical properties of cubic and rhombohedral GeTe
}

\author{
David J. Singh ${ }^{1}$ \\ Materials Science and Technology Division, Oak Ridge National Laboratory, \\ Oak Ridge, Tennessee 37831-6056
}

(Dated: 26 May 2022)

Calculations of the optical properties of GeTe in the cubic $\mathrm{NaCl}$ and rhombohedral ferroelectric structures are reported. The rhombohedral ferroelectric distortion increases the band gap from $0.11 \mathrm{eV}$ to $0.38 \mathrm{eV}$. Remarkably, substantial changes in optical properties are found even at high energies up to $5 \mathrm{eV}$. The results are discussed in relation to the bonding of GeTe and to phase change materials based on it.

PACS numbers: 78.20.Ci,71.20.Nr,64.70.ph 


\section{INTRODUCTION}

GeTe is a well known ferroelectric material with a Curie temperature $T_{C}$ of $\sim 670 \mathrm{~K}$ depending strongly on sample quality: $\underline{\underline{1}-\underline{\underline{6}}}$ The related compound, SnTe, is a low temperature ferroelectric again with strong sensitivity to sample quality $\underline{\underline{6}} \underline{\underline{7}} \mathrm{PbTe}$ is cubic but near a fer-

roelectric instability, which may be important in relation to its thermoelectric properties. $\underline{8} .9$ Above $T_{C}$, GeTe has a cubic $\mathrm{NaCl}$ type crystal structure, which undergoes a distortion consisting of a relative displacement of the Ge and Te sub-lattices along [111] accompanied by a sizable rhombohedral strain when cooled through $T_{C}$. At low temperatures this strain amounts to $\sim 2^{\circ}$. The driving mechanism for this instability was discussed by Lucovsky and White 10 and by Littlewood ${ }^{5}$ in terms of bonding, specifically the presence of p-electron bonding between Ge and Te, though from different perspectives. Lucovsky and White coined the term "resonance bonding" for this type of material, where the high symmetry crystal structure is not well adapted to the covalent bonding pattern of the elements and the result is a superposition of different covalent bond networks..$\underline{10}$

In any case, GeTe has been the subject of renewed interest due to its relationship with phase change materials used in data storage. $\stackrel{11}{-14}$ The ferroelectricity, as mentioned, is sensitive to disorder. When alloyed with $\mathrm{Sb}_{2} \mathrm{Te}_{3}$, the material adopts a cubic $\mathrm{NaCl}$ type crystalline structure in which one sub-lattice contains Te and the other contains an approximately charge balanced mixture of $\mathrm{Ge}, \mathrm{Sb}$ and vacancies, such that there is ideally one compensating vacancy $(V)$ for each two Sb atoms in the alloy, i.e. an approximate formula $\left(\mathrm{Ge}_{1-3 x} \mathrm{Sb}_{2 x} V_{x}\right)$ Te. Recently developed optical media (e.g. "blu-ray") use relatively low concentrations of $\mathrm{Sb}$, in some cases less than $10 \%$ (a typical composition is $\mathrm{Ge}_{8} \mathrm{Sb}_{2} \mathrm{Te}_{11}$ )! ${ }^{15}$

The $\mathrm{NaCl}$ structure crystalline state is either metallic or has a low band gap, $\sim 0.1 \mathrm{eV}$ or less depending on the detailed composition. Rapid quenching from the melt, however, yields a dense amorphous phase with a wider gap $\sim 0.5 \mathrm{eV}$ and rather different properties, specifically a lower bulk reflectivity and much higher resistivity. This amorphous phase is highly unusual in that it can be extremely rapidly converted to the crystalline phase by annealing, with reported time scales of $10-100$ ns (20 ns is typical in practical application). Furthermore, even shorter crystallization times may be possible with selected starting conditions. $\underline{16}$ While practical materials involve alloying with $\mathrm{Sb}$ or other modifications, similar behavior is also found in nominally pure GeTe films, including an amorphous phase with lower bulk 
reflectivity than the crystalline phase and rapid, sub-100 ns crystallization.

Here we report calculations of the optical properties of cubic and rhombohedral ferroelectric GeTe. As may be expected there is a change in the band gap between these states, amounting to $\sim 0.3 \mathrm{eV}$. In addition, we find that there is a substantial change in optical properties, specifically a strong decrease in reflectivity over a wide energy range up to almost $5 \mathrm{eV}$, or almost 15 times the band gap. This is a consequence of the role of covalent bonding in the ferroelectric instability of GeTe. We discuss the results in the context of phase change materials. Specifically, based on the results and consideration of existing experimental data we argue that there may be a relationship between the amorphous phase and the ferroelectric phase, and in particular that the amorphous phase may be a form of relaxor ferroelectric, with implications for the properties of the amorphous phase.

\section{COMPUTATIONAL APPROACH}

The calculations were performed within density functional theory with a modified exchange correlation potential designed to reproduce band gaps. They were done using the gen-

eral potential linearized augmented planewave (LAPW) method with local orbitals, $, 17,18$ as implemented in the WIEN2k code. ${ }^{19}$ LAPW sphere radii of 2.5 bohr and 2.6 bohr were used for Ge and Te, respectively, and spin-orbit was included, as is needed for this compound. ${ }^{20,21}$ We employed experimental crystal structures, i.e. a cubic lattice parameter of $a=5.976 \AA$, and rhombohedral ferroelectric structure, $a=4.16 \AA, c=10.68 \AA, u=0.4752$ (Ge at $(0,0,0)$; Te at $(0,0, u)$, in hexagonal coordinates, where $u=0.5$ is the ideal value for the $\mathrm{NaCl}$ structure and $u=0.25$ would correspond to a zinc blende tetrahedral structure).

The optical properties were obtained using the optical package of the WIEN2k code, which uses direct calculation of dipole matrix elements. Importantly, the modified BeckeJohnson potential developed by Tran and Blaha, denoted TB-mBJ was employed. ${ }^{22}$ This potential functional generally yields much improved band gaps for simple semiconductors and insulators relative to standard density functionals, $\stackrel{22-25}{2}$ which are designed to reproduce total energies and do not generally yield band gaps in reasonable accord with experiment. ${ }^{26}$ This approach was used previously to calculate optical properties of $\mathrm{PbTe}$ and $\mathrm{PbSe}$, which were found to be in good accord with experimental data, including the $1 \mathrm{eV}-5 \mathrm{eV}$ energy range, $\stackrel{27}{2}$ which is of importance here. Calculations for $\mathrm{Bi}_{4} \mathrm{Ti}_{3} \mathrm{O}_{12}$ also showed quantitative 
agreement with experiment..$^{28}$

\section{ELECTRONIC STRUCTURE AND OPTICAL PROPERTIES}

The relativistic band structure of cubic GeTe has been reported by many authors, starting with Herman and co-workers and Tung and Cohen. 20,21 Our result is similar to prior reports except for the exact value of the band gap reflecting the use of the TB-mBJ potential in the present calculations. Our calculated gap is $0.11 \mathrm{eV}$, as shown in Fig. 11 and is direct at the $\mathrm{L}$ point of the fcc zone in accord with prior work. This is also in accord with experimental measurements on $\mathrm{NaCl}$ structure (heavily $p$-type non-ferroelectric) films, which show a band gap in the range $0.1 \mathrm{eV}-0.2 \mathrm{eV} .29-31$ As an aside, we note that this band structure is qualitatively similar to that of PbTe and $\mathrm{PbSe}, \frac{32,33}{2}$ and is favorable for thermoelectric performance.

Turning to the rhombohedral ferroelectric structure, according to our calculations, the material has an indirect gap of $0.38 \mathrm{eV}$ in this state, while the direct gap (which remains at an $\mathrm{L}$ point) is $0.53 \mathrm{eV}$. The indirect gap in the ferroelectric state was previously noted by Yamanaka and co-workers $\stackrel{34}{ }$, and by Park and co-workers. $\stackrel{35}{\underline{2}}$ While this band gap change is large on a relative scale because of the small band gap of the cubic phase, it is far below the energy range where reflectivity changes are seen in phase change materials. For example, a typical laser probe for "blu-ray" media is at $405 \mathrm{~nm}(3.06 \mathrm{eV})$. The phase change materials used in such media show are sizable differences in optical properties between the $\mathrm{NaCl}$ and amorphous structures in this energy range.

The calculated bulk optical reflectivity is shown in Fig. 2 (note that the reflectivity of a thin film includes also contributions, which can be large, from the bottom surface and multiple reflections depending on the details of the film thickness and substrate; also the bulk reflectivity is an upper bound for a bulk sample, as for a real sample there will also be scattering, e.g. due to surface roughness or inhomogeneity, which will be wavelength dependent and will reduce the measured reflectivity). At $3 \mathrm{eV}$, the calculated direction averaged reflectivity is 0.68 for the ferroelectric phase and 0.77 for the cubic phase, amounting to a surprisingly large difference of $13 \%$.

Park and co-workers reported a detailed study of the optical properties of GeTe, $\mathrm{Sb}_{2} \mathrm{Te}_{3}$ and alloys using ellipsometry measurements on films as well as density functional 

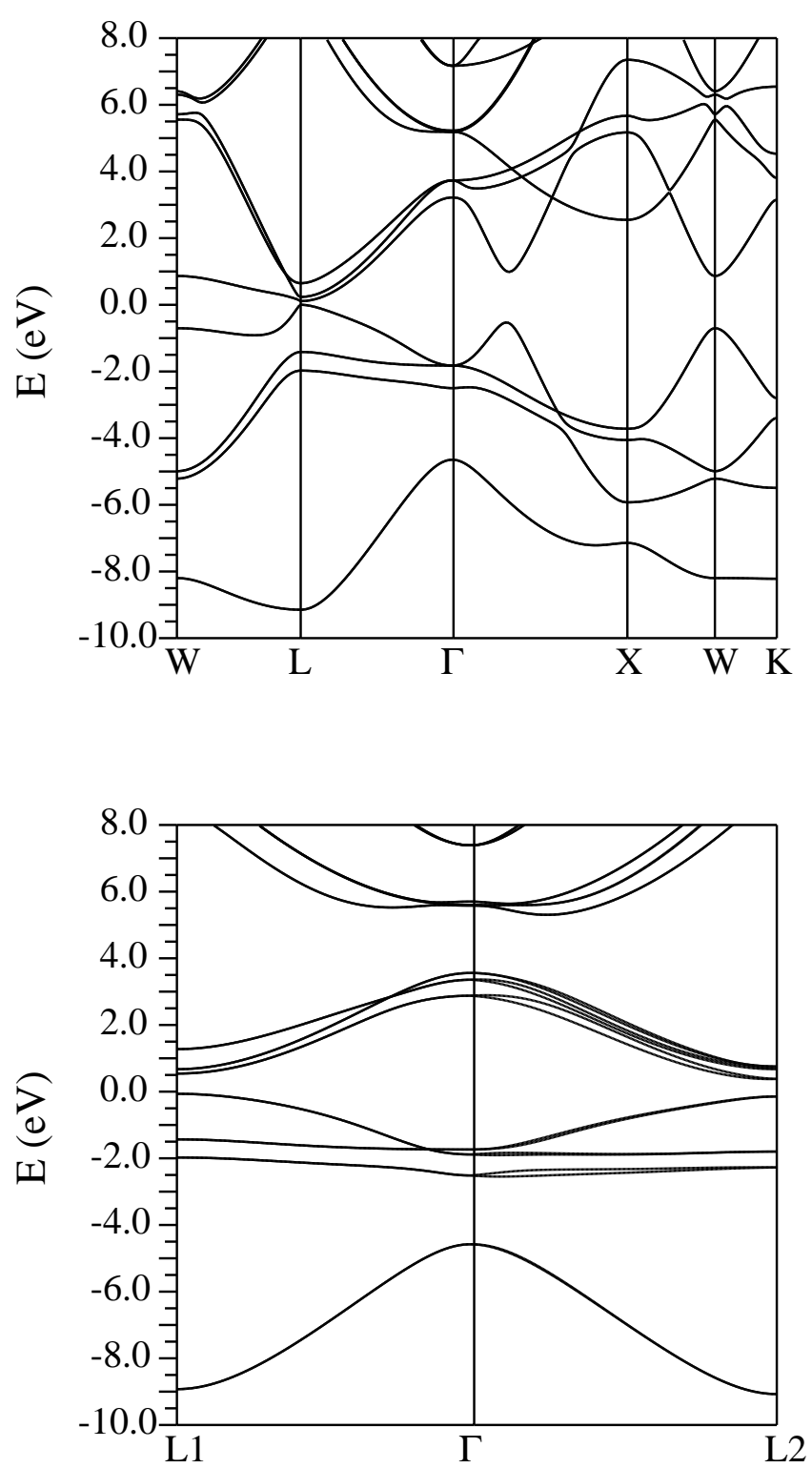

FIG. 1. Calculated band structure of cubic GeTe (top) and rhombohedral ferroelectric GeTe showing the non-equivalent $\Gamma$-L directions (bottom). The points "L1" and "L2" are the fcc L points along the rhombohedral $c$-axis and non- $c$-axis directions,respectively. Note the differences between these two directions, e.g. in the dispersion of the lowest conduction band from $\Gamma$. The direct band gaps at "L1" and "L2" are $0.61 \mathrm{eV}$ and $0.53 \mathrm{eV}$ respectively, while the indirect gap from the valence band at "L1" to the conduction band at "L2" is $0.45 \mathrm{eV}$. The lowest indirect gap is from a valence band away from a symmetry point approximately along a line joining an "L1" to an "L2" point to the "L2" point conduction band and is $0.38 \mathrm{eV}$. 


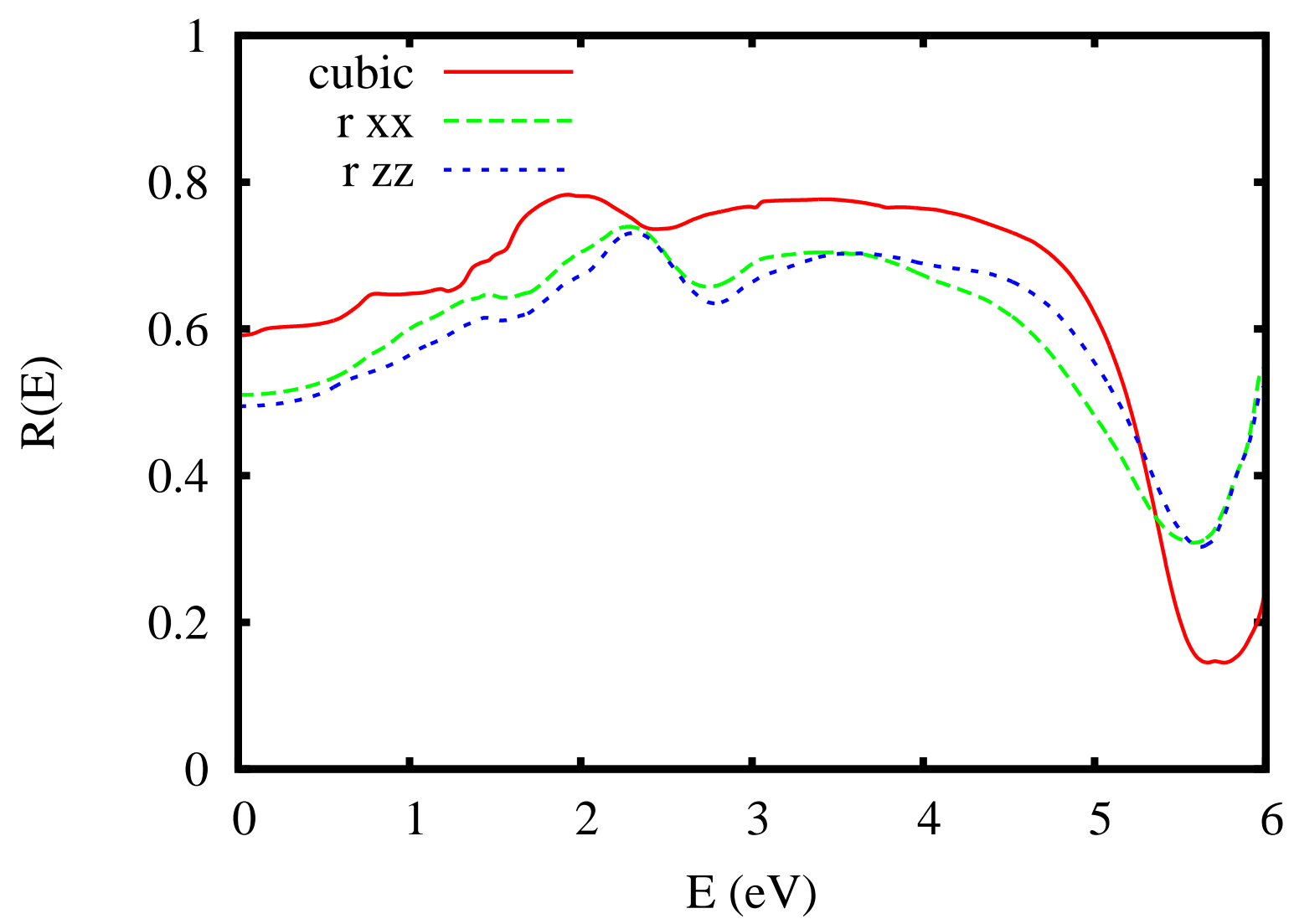

FIG. 2. Calculated optical reflection for cubic and rhombohedral ferroelectric GeTe. For the rhombohedral case, "zz" denotes polarization along the ferroelectric polar axis, while "xx" denotes in polarization perpendicular to it.

calculations. $\frac{35}{5}$ Yamanaka and co-workers ${ }^{34}$ presented density functional calculations of optical constants of $\mathrm{GeTe}$ and $\mathrm{Ge}_{2} \mathrm{Sb}_{2} \mathrm{Te}_{5}$ using the local density approximation along with experimental measurements of optical constants. Optical reflectivity spectra of GeTe over a wide energy range were reported by Cardona and Greenway on polycrystalline GeTe films. $\underline{36}^{3}$ While the measured absolute reflectivity is consistently lower than the calculated values, perhaps because of scattering in the non-single crystal film, similar structures are seen, in particular a maximum at $\sim 2 \mathrm{eV}$, and a minimum just above $5 \mathrm{eV}$. In the data of Cardona and Greenway there is a small maximum above this latter minimum, while we obtain a larger maximum. In both the experimental data and the calculated spectrum there is a smooth fall-off in reflectivity at energies above this. The low energy (where off-specular scattering should be less important) extrapolated experimental reflectivity is 0.5 as compared to the calculated value 0.59 for cubic GeTe and a direction averaged 0.5 for ferroelectric, which 
would constitute good agreement assuming that the film measured was ferroelectric.

As mentioned, the driving force for the ferroelectric transition in GeTe has been discussed in terms of $p$-electron bonding. In the resonant bond picture of Lucovsky and White, 10 electrons in bonding orbitals are shared between different bonds and both the cubic and ferroelectric states are substantially covalent (it should be noted that sharing electrons in a bonding network would normally stabilize a highly covalent high symmetry state as in aromatic rings and multicenter bonds). The conclusion that p-electron bonding is important for the ferroelectricity has also been reached using first principles calculations, though the details of the bonding are different. $\underline{.37}$ Interestingly, there is a substantial strain coupling between the frozen-in zone center transverse optic mode and the rhombohedral strain, ${ }^{38}$ presumably as a consequence of the covalent bonding in the ferroelectric state.

Fig. 3 shows the calculated projections of the electronic density of states of Ge and Te $p$-character in the LAPW spheres. This is a quantity that is proportional to the $p$ contributions to the electronic structure. As may be seen, both the valence and conduction band regions consist of mixtures of Ge $p$ and Te $p$ states, similar to what has been shown in prior studies. $\stackrel{34,37}{ }$ Descriptions of bonding in solids are necessarily qualitative because of the ambiguity of assigning charge and orbital characters to different sites. Nonetheless, it is apparent from the figure that the nature of the states in the conduction band is quite different from those in the valence band, even at the band edges, in contrast to a simple interpretation of resonant bonds.

Specifically, the valence bands have relatively much higher Te $p$ character than the conduction bands, and conversely for the Ge $p$ character. This is in contradiction to what is anticipated for a resonant bonded configuration, and is more like what is expected for an ionic material, consisting nominally of $\mathrm{Ge}^{2+}$ and $\mathrm{Te}^{2-}$ ions, with some covalent character arising from cross gap hybridization between $p$-orbitals on the anion and cation sites. This is consistent both with the octahedral coordinated $\mathrm{NaCl}$ type crystal structure, which is an ionic crystal structure, as well as with the results of Waghmare and co-workers, $\underline{37}$ who found highly enhanced Born effective charges, $Z_{\mathrm{Ge}}^{*}=-Z_{\mathrm{Te}}^{*}=10.8$, as compared to the nominal valence of 2.0. Such large enhancements of the Born effective charge are characteristic of conventional ferroelectrics such as $\mathrm{BaTiO}_{3}$, and arise from cross-gap hybridization between nominally occupied states on the anion $(\mathrm{O})$ and unoccupied states on the active cation (Ti) $\underline{\underline{39}-41}$ In contrast, the Born effective charges of covalent semiconductors are generally 


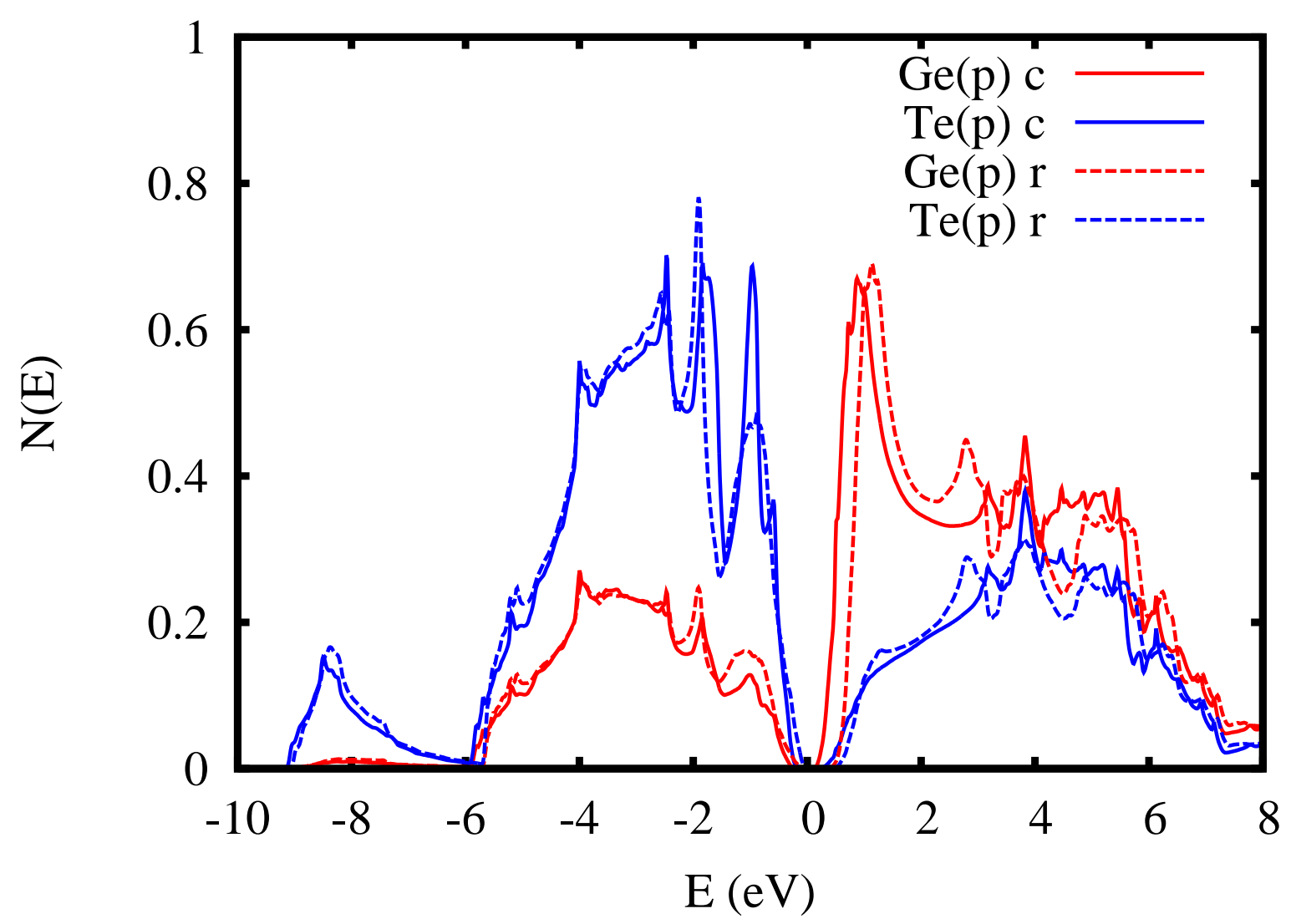

FIG. 3. Calculated p-character projections of the electronic density of states of $\mathrm{NaCl}$ cubic (c) and rhombohedral (r) GeTe onto the Ge and Te LAPW spheres. The energy zero is set to the valence band maximum.

reduced from the nominal valences.

Cross-gap hybridization is understood as a main driving force for ferroelectricity in conventional oxides such as $\mathrm{BaTiO}_{3}, \stackrel{40,41}{,}$ where the enhanced Born charges reflect an improved bonding with distortion. Similarly, the ferroelectricity of GeTe may be regarded as arising from the instability of an ionic cubic structure due to covalency between anions and cations. In view of this mechanism, one expects changes in the density of states particularly corresponding to the orbitals involved with the ferroelectric distortion. In fact, this can be seen in Fig. 3, though rather weakly because of the small displacement of the atoms. Besides the upward energy shift of the conduction band edge in the ferroelectric state, one notes an increased $p$ contribution for the ferroelectric state in the energy range near $3 \mathrm{eV}$ and a decrease in the vicinity of $4 \mathrm{eV}-5 \mathrm{eV}$. This is accompanied by a small downward shift of the density of states in the valence bands in the range near $-2 \mathrm{eV}$. While these changes are 
small they underlie the change in optical reflectivity at energies well above the band gap. In short, the fact that the ferroelectric instability is connected to the $p$-bonding results in large changes in reflectivity between the cubic and ferroelectric structures.

\section{DISCUSSION AND CONCLUSIONS}

This may have implications for understanding the phase change materials. Experimental investigation and first principles structural studies and molecular dynamics have shown that the local structure of the amorphous phase of $\mathrm{GeTe}_{-} \mathrm{Sb}_{2} \mathrm{Te}_{3}$ alloys is more characteristic of a $p$-bonded covalent material than the cubic structure. $\underline{42-55}$ One complication is that the amorphous phase is not an equilibrium thermodynamic phase but is a frozen in structure, which in principle depends on the sample history. In any case, most studies have focused on the composition $\mathrm{Ge}_{2} \mathrm{Sb}_{2} \mathrm{Te}_{5}$, which has $20 \%$ vacancies on the cation site. ${ }^{56}$ Not surprisingly, there are distortions around the vacancies leading to low coordinated Te atoms, while other Te atoms retain octahedral coordination. $\underline{42,43,45}$ Recent work shows an important role for the vacancy ordering in determining the electrical resistivity. $\underline{55}$ While all the coordinations are changed in the amorphous structures, detailed studies indicate the presence of both of large numbers of approximately octahedral coordinated atoms, $\stackrel{44}{,}$ as in the $\mathrm{NaCl}$ structure and the ferroelectric structure of GeTe, as well as some, possibly small, $, \underline{46}, \underline{0}$ fraction of approximately tetrahedral sites for Ge. $\stackrel{43,49}{.}$

What the present results show is that just the relatively subtle structural changes associated with ferroelectricity can lead to large changes in optical properties. Based on this, one can speculate that this may be an important aspect of the structure of the amorphous phase as it relates to optical reflectivity. We observe that the crystallization temperatures of $\sim 450 \mathrm{~K}-600 \mathrm{~K}$ are comparable to the ferroelectric Curie temperature of GeTe. In general, long range ferroelectric order can be suppressed by either disorder or doping. Disorder in particular can preserve the local chemical environment that favors off-centering. It may be that the amorphous state, characterized by disordered, locally inhomogeneous vacancies, has characteristics of a relaxor ferroelectric, while the less disordered crystalline phase is more similar to the non-ferroelectric cubic state.

Relaxor ferroelectrics, which can be produced from standard ferroelectrics by strong disorder, are characterized by a Burns temperature, $T_{B}$, below which the local structure 
becomes similar to a ferroelectric, while the long range symmetry does not $\underline{57}, \underline{58}$ Relaxor ferroelectrics do not show long range ferroelectric order, but do show characteristics of the ferroelectric phase. In the present case, because of the existence of nanoscale polar regions (so called polar nanoregions, or PNR) in relaxor materials below $T_{B}$, one may expect optical properties similar to the ferroelectric phase. Besides their ferroelectric like local structures,

characteristics of relaxor ferroelectrics are an enhanced dielectric response in the form of a prominent broad peak in the temperature dependent static dielectric constant, $\epsilon_{0}$, along with a characteristic frequency dependence of the peak. We suggest that it will be of interest to study the dielectric behavior and other properties of the amorphous phase to determine whether signatures of relaxor ferroelectricity are present. This may provide new insight into the structure of the amorphous phase and additionally may potentially have implications for the electrical properties of devices based on electrical sensing such as phase change random access memories.

\section{ACKNOWLEDGMENTS}

I am grateful for helpful discussions with R.O. Jones and S.R. Elliott. Work at ORNL was supported by the Department of Energy, Basic Energy Sciences, Materials Sciences and Engineering Division.

\section{REFERENCES}

${ }^{1}$ K. Schubert and H. Fricke, Z. Naturforsch. A 6, 781 (1951).

${ }^{2}$ K. Schubert and H. Fricke, Z. Metallkunde 44, 457 (1953).

${ }^{3}$ J. N. Bierly, L. Muldawaer, and O. Beckman, Acta Metallurgica 11, 447 (1963).

${ }^{4}$ J. Goldak, C. S. Barrett, D. Innes, and W. Youdelis, J. Chem. Phys. 44, 3323 (1966).

${ }^{5}$ P. B. Littlewood, J. Phys. C: Solid St. Phys. 13, 4855 (1980).

${ }^{6}$ G. S. Pawley, W. Cochran, R. A. Cowley, and G. Dolling, Phys. Rev. Lett. 17, 753 (1966).

${ }^{7}$ M. IIzumi, Y. Hamaguchi, K. F. Komatsubara, and Y. Kato, J. Phys. Soc. Japan 38, 443 (1973).

${ }^{8}$ J. An, A. Subedi, and D. J. Singh, Solid State Commun. 148, 417 (2008). 
${ }^{9}$ O. Delaire, J. Ma, K. Marty, A. F. May, M. A. McGuire, M. H. Du, D. J. Singh, A. Podlesnyak, G. Ehlers, M. D. Lumsden, and B. C. Sales, Nature Materials 10, 614 (2011).

${ }^{10}$ G. Lucovsky and R. M. White, Phys. Rev. B 8, 660 (1973).

${ }^{11}$ N. Yamada, E. Ohno, N. Akahira, K. Nishiuchi, K. Nagata, and M. Takao, Jpn. J. Appl. Phys. 26, 61 (1987).

${ }^{12}$ N. Yamada, E. Ohno, K. Nishiuchi, N. Akahira, and M. Takao, J. Appl. Phys. 69, 2849 (1991).

${ }^{13}$ M. Wuttig and N. Yamada, Nature Materials 6, 824 (2007).

${ }^{14}$ S. Raoux, Ann. Rev. Mater. Res. 39, 25 (2009).

${ }^{15}$ N. Yamada, R. Kojima, M. Uno, T. Akiyama, H. Kitaura, K. Narumi, and K. Nishiuchi, Proc. SPIE 4342, 55 (2002).

${ }^{16}$ D. Loke, T. H. Lee, W. J. Wang, L. P. Shi, R. Zhao, Y. C. Yeo, T. C. Chong, and S. R. Elliott, Science 336, 1566 (2012).

${ }^{17}$ D. Singh, Phys. Rev. B 43, 6388 (1991).

${ }^{18}$ D. J. Singh and L. Nordstrom, Planewaves Pseudopotentials and the LAPW Method, 2nd Edition (Springer, Berlin, 2006).

${ }^{19}$ P. Blaha, K. Schwarz, G. Madsen, D. Kvasnicka, and J. Luitz, WIEN2k, An Augmented Plane Wave + Local Orbitals Program for Calculating Crystal Properties (K. Schwarz, Tech. Univ. Wien, Austria) (2001).

${ }^{20}$ F. Herman, R. L. Kortum, R. B. Ortenburger, and J. P. Van Dyke, J. Phys. (Paris) C4, 62 (1968).

${ }^{21}$ Y. W. Tung and M. L. Cohen, Phys. Rev. 180, 823 (1969).

${ }^{22}$ F. Tran and P. Blaha, Phys. Rev. Lett. 102, 226401 (2009).

${ }^{23}$ D. J. Singh, Phys. Rev. B 82, 205102 (2010).

${ }^{24}$ D. Koller, F. Tran, and P. Blaha, Phys. Rev. B 83, 195134 (2011).

${ }^{25}$ D. J. Singh, Phys. Rev. B 82, 155145 (2010).

${ }^{26}$ J. P. Perdew, J. A. Chevary, S. H. Vosko, K. A. Jackson, M. R. Pederson, D. J. Singh, and C. Fiolhais, Phys. Rev. B 46, 6671 (1992).

${ }^{27}$ C. E. Ekuma, D. J. Singh, J. Moreno, and M. Jarrell, Phys. Rev. B 85 (2012).

${ }^{28}$ D. J. Singh, S. S. A. Seo, and H. N. Lee, Phys. Rev. B 82, 180103 (2010).

${ }^{29}$ R. Tsu, W. E. Howard, and L. Esaki, Solid State Commun. 5, 167 (1967). 
${ }^{30}$ R. Tsu, W. E. Howard, and L. Esaki, Phys. Rev. 122, 779 (1968).

${ }^{31}$ S. K. Bahl and K. L. Chopra, J. Appl. Phys. 41, 2196 (1970).

${ }^{32}$ D. J. Singh, Phys. Rev. B 81, 195217 (2010).

${ }^{33}$ D. Parker and D. J. Singh, Phys. Rev. B 82, 035204 (2010).

${ }^{34}$ S. Yamanaka, S. Ogawa, I. Morimoto, and Y. Ueshima, Jpn. J. Appl. Phys. 37, 3327 (1998).

${ }^{35}$ J. W. Park, S. H. Eom, H. Lee, J. L. F. Da Silva, Y. S. Kang, T. Y. Lee, and Y. H. Khang, Phys. Rev. B 80, 115209 (2009).

${ }^{36}$ M. Cardona and D. L. Greenway, Phys. Rev. 133, A1685 (1964).

${ }^{37}$ U. V. Waghmare, N. A. Spaldin, H. C. Kandpal, and R. Seshardi, Phys. Rev. B 67, 125111 (2003).

${ }^{38}$ K. M. Rabe and J. D. Joannopoulos, Phys. Rev. B 36, 6631 (1987).

${ }^{39}$ J. D. Axe, Phys. Rev. 157, 429 (1967).

${ }^{40}$ R. E. Cohen, Nature (London) 358, 136 (1992).

${ }^{41}$ W. Zhong, R. D. King-Smith, and D. Vanderbilt, Phys. Rev. Lett. 72, 3618 (1994).

${ }^{42}$ S. Caravati, M. Bernasconi, T. D. Kuhne, M. Krack, and M. Parrinello, Appl. Phys. Lett. 91, 171906 (2007).

${ }^{43}$ J. Akola and R. O. Jones, Phys. Rev. B 76, 235201 (2007).

${ }^{44}$ J. Hegedus and S. R. Elliott, Nature Materials 7, 399 (2008).

${ }^{45}$ Z. Sun, J. Zhou, A. Blomqvist, B. Johansson, and R. Ahuja, Phys. Rev. Lett. 102, 075504 (2009).

${ }^{46}$ J. L. F. Da Silva, A. Walsh, S. H. Wei, and H. Lee, J. Appl. Phys. 106, 113509 (2009).

${ }^{47}$ M. Xu, Y. Q. Cheng, H. W. Sheng, and E. Ma, Phys. Rev. Lett. 103, 195502 (2009).

${ }^{48}$ J. Akola and R. O. Jones, Phys. Status Solidi B 249, 1851 (2012).

${ }^{49}$ X. Q. Liu, X. B. Li, L. Zhang, Y. Q. Cheng, Z. G. Yan, M. Xu, X. D. Han, S. B. Zhang, Z. Zhang, and E. Ma, Phys. Rev. Lett. 106, 025501 (2011).

${ }^{50}$ J. Y. Raty, C. Bichara, R. Mazzarello, P. Rausch, P. Zalden, and M. Wuttig, Phys. Rev. Lett. 108, 239601 (2012).

${ }^{51}$ M. Krbal, A. V. Kolobov, P. Fons, J. Tominaga, S. R. Elliott, J. Hegedus, T. Matsunaga, N. Yamada, K. Nitta, and T. Uruga, Phys. Rev. B 86, 045212 (2012).

${ }^{52}$ J. Kalikka, J. Akola, J. Larrucea, and R. O. Jones, Phys. Rev. B 86, 144113 (2012).

${ }^{53}$ T. H. Lee and S. R. Elliott, Phys. Status Solidi B 249, 1886 (2012). 
${ }^{54}$ J. M. Skelton, T. H. Lee, and S. R. Elliott, Appl. Phys. Lett. 101, 024106 (2012).

${ }^{55}$ W. Zhang, A. Theiss, P. Zalden, R. Zeller, P. H. Dederichs, J. Y. Raty, M. Wuttig, S. Blugel, and R. Mazzarello, Nature Materials 11, 952 (2012).

${ }^{56}$ T. Nonaka, G. Ohbayashi, Y. Toriumi, Y. Mori, and H. Hashimoto, Thin Solid Films 370, 258 (2000).

${ }^{57}$ A. A. Bokov and Z. G. Ye, J. Mater. Sci. 41, 31 (2006).

${ }^{58}$ T. Egami, Ann. Rev. Mater. Res. 37, 297 (2007). 